\title{
INOVAFEI E OS TRABALHOS DE CONCLUSÃO DE CURSO DE ENGENHARIA QUÍMICA DO CENTRO UNIVERSITÁRIO DA FEI
}

\author{
R. B. TÔRRES ${ }^{1}$, I. C. FRANCO ${ }^{1}$, L. F. NOVAZZI ${ }^{1, *}$ \\ ${ }^{1}$ Centro Universitário FEI, Departamento de Engenharia Química \\ *E-mail: lnovazzi@fei.edu.br
}

\begin{abstract}
RESUMO: O INOVAFEI foi instituído no Centro Universitário da FEI em 2013 e é um evento semestral no qual são expostos os Trabalhos de Conclusão de Curso de diversos cursos da escola. Esses trabalhos de conclusão são desenvolvidos num período de um ano e acompanhados por um professor orientador. A natureza desses trabalhos é a de integrar conhecimentos de Engenharia Química e áreas correlatas. Nesse artigo faz-se uma breve descrição envolvendo a condução dos Trabalhos de Conclusão de Curso na Engenharia Química, destacando-se a realização dos trabalhos não individualmente, mas em grupos de alunos, a fim de se desenvolver o espírito de equipe. Um professor avaliador acompanha um dado grupo desde a proposta inicial do trabalho até a versão final do projeto, com o intuito de verificar sua evolução. Desde a criação do INOVAFEI foram executados 51 trabalhos, principalmente na área ambiental e na de tecnologias limpas. Os dois melhores trabalhos do INOVAFEI de cada semestre são premiados, promovendo um ambiente de competição saudável entre os estudantes.
\end{abstract}

PALAVRAS-CHAVE: Trabalho de conclusão de curso; INOVAFEI; Engenharia Química; ensino.

\section{INTRODUÇÃO}

O INOVAFEI ou Exposição dos Trabalhos de Conclusão de Curso do Centro Universitário da FEI é um evento relativamente recente na instituição, tendo sido criado em dezembro de 2013. Foi uma forma de unificar a apresentação dos trabalhos de conclusão de curso (TCCs) de diferentes modalidades de Engenharia, englobando Civil, Elétrica, Automação e Controle, Produção, Química e Têxtil. Além dessas áreas, no INOVAFEI também são apresentados os projetos de formatura dos cursos de Ciência da Computação e de Administração. A ideia é que os graduandos mostrem seus trabalhos para um público bastante amplo, utilizando pôsteres e / ou protótipos. O evento é semestral e organizado por todos os departamentos de ensino pertinentes, juntamente com o setor de Comunicação e Marketing. Há a participação de pelo menos 150 estudantes e os melhores trabalhos, avaliados por profissionais da academia e do meio industrial, são premiados. Para ilustrar o que foi comentado nesse parágrafo, indica-se na Figura 1 uma foto do último INOVAFEI, 
realizado em dezembro de 2017.

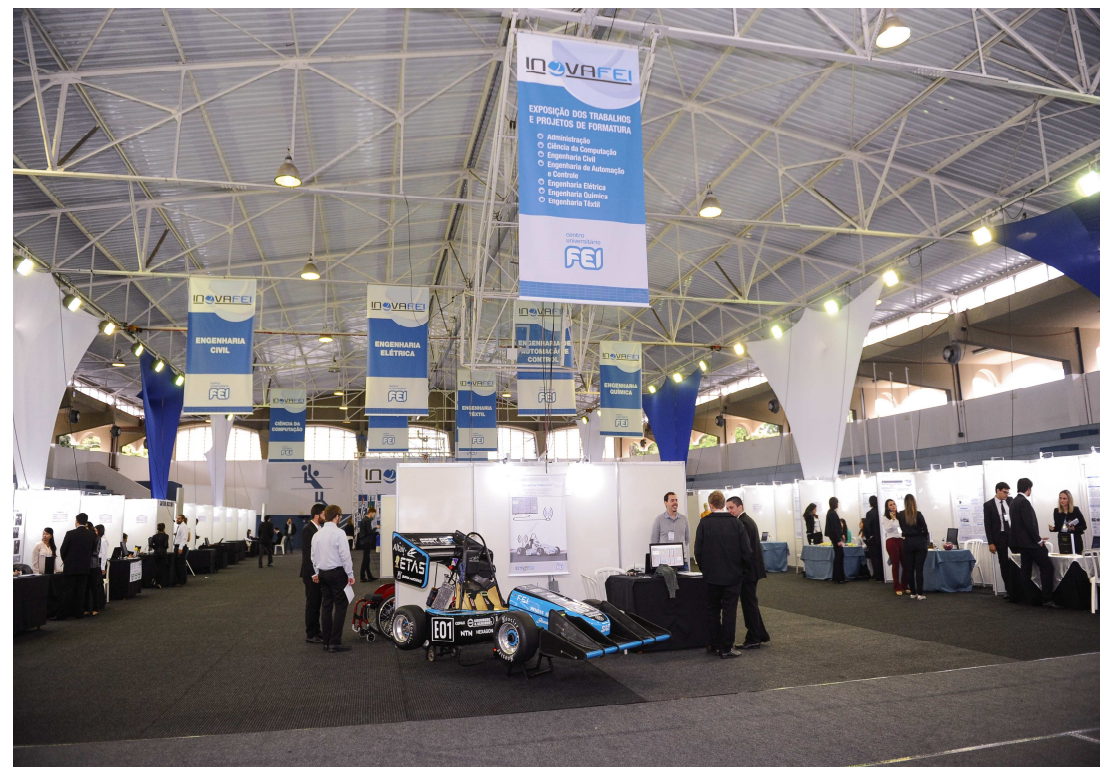

Figura 1 - INOVAFEI 2017.

O evento é realizado no Ginásio de Esportes da instituição e a distribuição dos diversos trabalhos é mostrada na Figura 2, com uma área disponível de mais de $1000 \mathrm{~m}^{2}$. Pode-se notar que a parte central direita do ginásio é ocupada por projetos da Engenharia Elétrica, que se subdivide nas ênfases de Eletrônica, Computadores e Telecomunicações. Isso se deve ao fato que os trabalhos dessa área são apresentados por meio de protótipos, como se observa pelo veículo elétrico retratado na Figura 1. Ainda na parte central do ginásio há os projetos de Engenharia Têxtil e Administração, enquanto que as laterais da exposição, junto às arquibancadas, são ocupadas pelos trabalhos de Engenharia Química, Engenharia Civil e Ciência da Computação. A apresentação dos projetos de Engenharia Química é feita por meio de pôsteres e também protótipos, dependendo da natureza do trabalho.

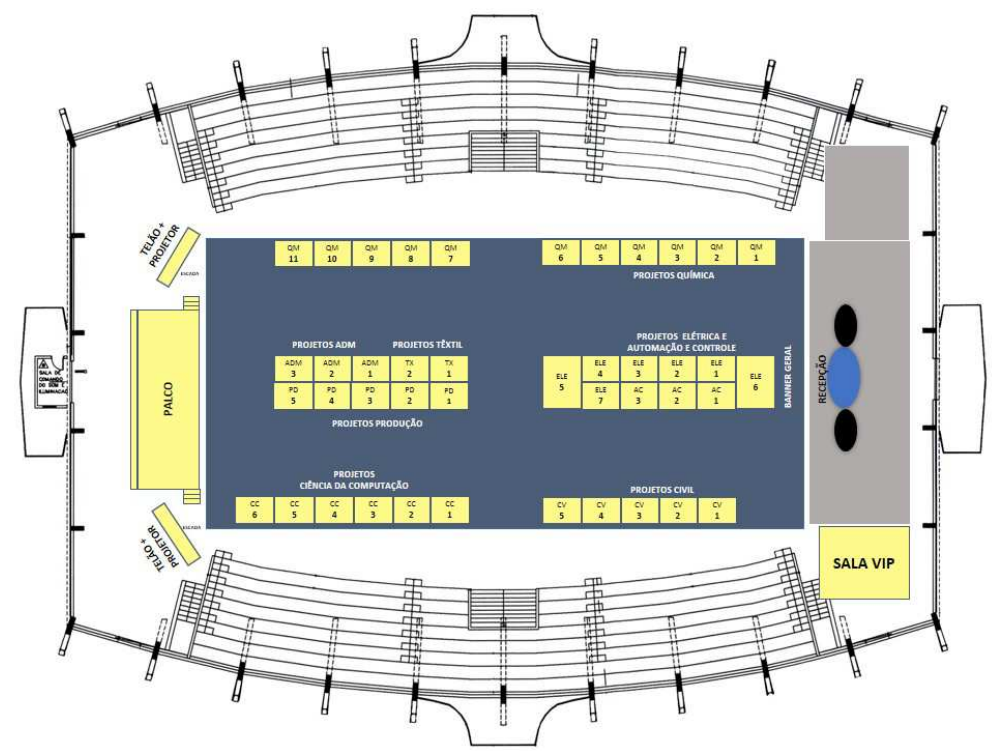

Figura 2 - Distribuição dos trabalhos no INOVAFEI. 
Além de uma breve descrição do evento, pretende-se nesse artigo realizar a discussão a respeito de como é a condução dos TCCs de Engenharia Química no Centro Universitário da FEI, desde a formação dos grupos de alunos até a apresentação final no INOVAFEI. Independentemente da modalidade de curso, do local e da natureza da instituição de ensino, espera-se que algumas das experiências aqui compartilhadas possam ser úteis para a melhoria de condições das disciplinas de TCCs ofertadas em cursos de Engenharia no Brasil. Nessa edição especial da revista The Journal of Engineering and Exact Sciences, o leitor poderá verificar que os artigos resultantes dos melhores TCCs do curso de Engenharia Química possuem qualidade bastante aceitável, tendo-se em conta que são resultantes de trabalhos de graduação. Isso é um indicativo que a realização desses TCCs tem sido, no aspecto geral, positiva.

\section{O TCC DE ENGENHARIA QUÍMICA}

O Trabalho de Conclusão de Curso de Engenharia Química no Centro Universitário da FEI é realizado num período de um ano, o que corresponde a disciplinas específicas do nono e décimo semestres, representadas de modo simplificado por TCC1 e TCC2, respectivamente. Isso significa que o estudante matriculado nessas disciplinas é quase um engenheiro formado e carrega consigo a bagagem imprescindível de Fenômenos de Transporte, Termodinâmica, Reatores Químicos e Operações Unitárias, dentre várias outras ferramentas. Um dos objetivos primordiais do TCC é promover a integração e aplicação desses conhecimentos. Para reforçar essa ideia, cabe aqui reproduzir um trecho da resolução do Conselho Nacional de Educação e Câmara de Educação Superior CNE/CES 11/2002, que estabelece o seguinte no Artigo $5^{\circ}$ :

"Artigo 5": Cada curso de Engenharia deve possuir um projeto pedagógico que demonstre claramente como o conjunto das atividades previstas garantirá o perfil desejado de seu egresso e o desenvolvimento de competências e habilidades esperadas. Ênfase deve ser dada à necessidade de se reduzir o tempo em sala de aula, favorecendo o trabalho individual e em grupo dos estudantes.

$\S 1^{\circ}$ Deverão existir os trabalhos de síntese e integração dos conhecimentos adquiridos ao longo do curso, sendo que, pelo menos, um deles deverá se constituir em atividade obrigatória como requisito para a graduação."

Outra questão a ser abordada é sobre o TCC propriamente dito: afinal de contas, o que exatamente pode ser considerado um TCC? Há livros específicos que discutem o assunto, como os de Martins Jr. (2008) e Costa e Meira (2012), por exemplo. Há também um interessante artigo de d'Angelo et al. (2013), no qual se faz um apanhado das diversas formas de se encarar ou de se definir um Trabalho de Conclusão de Curso em algumas Instituições de Ensino Superior brasileiras. Esses autores também apresentam as experiências vividas no curso de Engenharia Química da Unicamp, mostrando pontos positivos e pontos negativos. Uma das conclusões desse trabalho de Campinas é que há quatro pontos em comum na implantação do Trabalho de Conclusão de Curso em quase qualquer curso de Engenharia, resumidos a seguir:

- A disciplina de TCC deve ser formal e fazer parte da grade curricular; 
- O tema de TCC deve ser decidido pelo aluno;

- O trabalho é orientado por um docente;

- O TCC deve ter caráter de síntese e integração de conhecimento.

Não existe muito o que se questionar sobre o primeiro ponto citado. A disciplina de TCC deve fazer parte da grade curricular e ser obrigatória: é nela que se faz o controle acadêmico de todas as atividades desenvolvidas. Além disso, essa disciplina também pode ser empregada para se discorrer sobre a estrutura de um trabalho formal. Por mais surpreendente que isso possa ser, há muitos estudantes quase formados e que ainda têm uma ideia muito vaga de tal estrutura. Outro quesito a ser explorado nesse tipo de disciplina tem a ver com a pesquisa bibliográfica. Os alunos precisam ter contato com diferentes bases de dados, revistas científicas, patentes, etc., ou seja, eles devem saber onde buscar informações técnicas. É a coisa mais comum hoje em dia um estudante querer resolver qualquer tipo de problema a partir de uma busca superficial e em poucos minutos na Internet. Mais uma vez, a Internet pode ser uma boa fonte de dados, mas é preciso saber o que e como se procurar.

O segundo tópico envolve o fato de o próprio aluno ou grupo de alunos escolher um tema de TCC. O aspecto mais positivo de tal abordagem é a possibilidade de o estudante exercer sua criatividade, não sendo limitado por temas previamente arranjados. Desse modo, muitos trabalhos podem ter viés empreendedor e serem embriões de pequenos negócios, com potencial de crescimento. Nesse contexto, vale a pena discutir e analisar alguns dos resultados provenientes de uma pesquisa feita em 2015 pelo Global Entrepeneurship Monitor (GEM), a pedido do Sebrae. Na Figura 3 são apresentadas as taxas de empreendedorismo em estágio inicial, com os países agrupados em função da fase de desenvolvimento econômico. Essa taxa representa a percentagem de empreendedores na população de 18 a 64 anos de cada país.

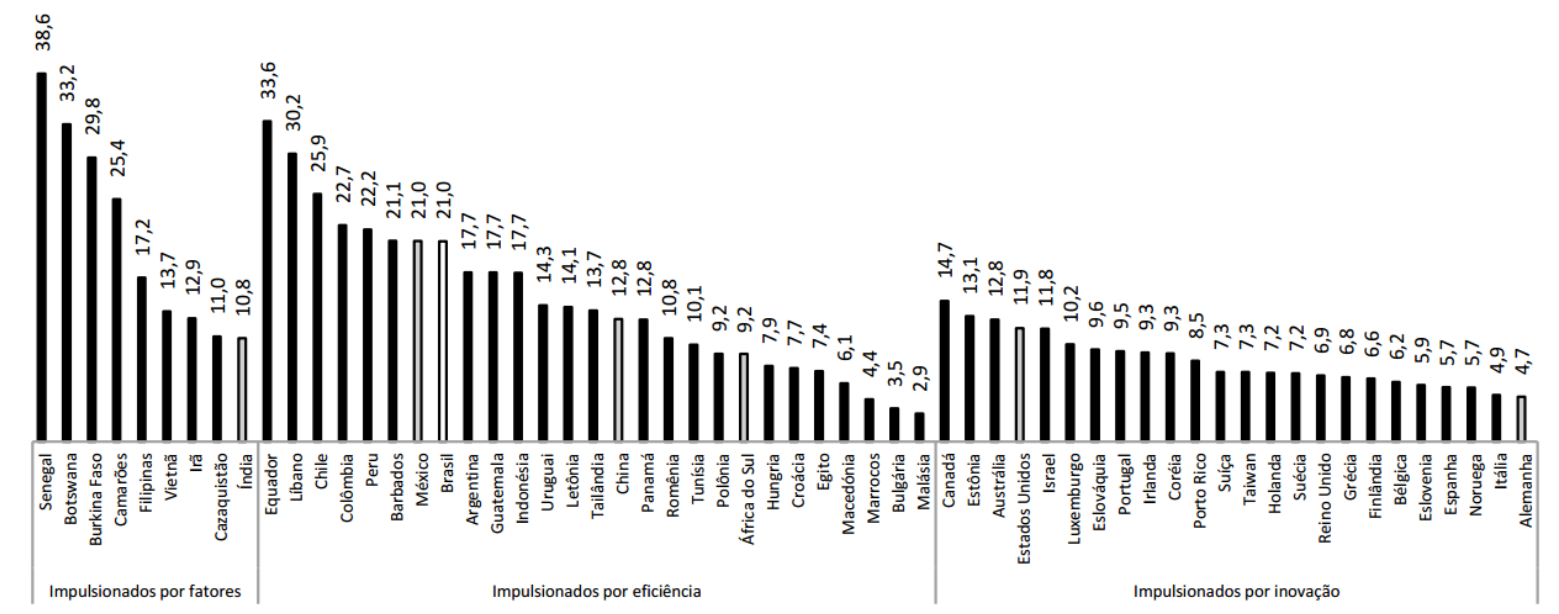

Figura 3 - Taxa de empreendedorismo em estágio inicial 2015.

O grupo de países impulsionados por eficiência, do qual o Brasil faz parte, compreende as nações caracterizadas pelos avanços na industrialização e ganhos de escala. Trata-se de uma característica típica da Engenharia Química, na qual modificações e melhorias nos processos podem levar a ganhos de produtividade. Já os países classificados como 
impulsionados por inovação são caracterizados por atividades intensivas em conhecimento e modernização do setor de serviços. Esse tipo de atividade também faz parte do escopo de Engenharia Química, como no desenvolvimento de um novo produto, por exemplo. Seja na impulsão por eficiência ou por inovação, o TCC pode ser um meio adequado para despertar jovens empreendedores. Esse tipo de ideia é fomentado pelos docentes da FEI que estão ligados com o TCC.

Nos TCCs de Engenharia Química do Centro Universitário da FEI, os alunos têm toda a liberdade na escolha do tema. Entretanto, é preciso se analisar o lado negativo dessa flexibilidade e se enfrentar a realidade. Muitos estudantes ainda não possuem suficiente maturidade para escolher um tema adequado. Nesse caso, é papel dos docentes responsáveis pela disciplina de TCC1 induzir e orientar os alunos na escolha. Em casos mais extremos, os professores podem inclusive propor um dado tema. Não se trata do ideal, claramente, mas sim daquilo que é possível nessas circunstâncias.

Ainda em relação à escolha de um tema, muitos grupos procuram buscar problemas ligados a indústrias nas quais estagiam. Isso é fortemente incentivado e por diversas razões. A primeira delas é que os estudantes lidam com uma questão real e devem propor uma solução viável para resolvê-la. A segunda é que isso pode abrir portas para os egressos no mercado de trabalho. A combinação desses dois motivos também permite um maior estreitamento entre o setor empresarial e o meio acadêmico, algo que em geral não é feito de modo sistemático em Instituições de Ensino Superior.

O terceiro ponto comum dos TCCs é que o projeto precisa ser orientado por um docente. Para essa afirmação, não parece haver nenhum argumento contrário. Salvo exceções, um aluno de graduação, mesmo que cursando o último ano, ainda não tem discernimento suficiente para conduzir por conta própria um Trabalho de Conclusão de Curso. É preciso que um professor seja responsável por esse processo. O quarto ponto comum nos TCCs diz respeito à síntese e à integração do conhecimento. Isso é da própria natureza do Trabalho de Conclusão de Curso e já foi justificado anteriormente.

Além desses quatro pontos comuns, há mais duas questões pertinentes à condução do TCC, como elencado a seguir:

- O TCC deveria ser individual ou em grupo?

- Como pode ser avaliado um TCC?

Normalmente, as Instituições de Ensino Superior atacam essas questões utilizando estratégias diferentes. A primeira tem a ver com o TCC ser individual ou em grupo. Quando ele é realizado individualmente, acaba aumentando bastante a responsabilidade do aluno. Entretanto, essa não é a política adotada nos TCCs do curso de Engenharia Química do Centro Universitário da FEI. Isso acontece porque a orientação de trabalhos individuais requer muitas horas de dedicação do corpo docente, o que pode ser bastante complicado tendo-se em vista as várias atividades em que os professores estão envolvidos em seu dia a dia.

Outro argumento que sustenta essa política está relacionado com o trabalho em grupo. 
É extremamente importante que os estudantes sejam capazes de trabalhar com seus colegas de turma, cumprindo prazos e atingindo metas. Deve-se lembrar que a formação do Engenheiro contempla não só a parte técnica, mas muitos critérios subjetivos e de personalidade. Desse modo, os TCCs de Engenharia Química são executados em grupos de 4 ou no máximo 5 alunos. Observou-se que esse número de componentes no grupo funciona bem: as tarefas do trabalho podem ser divididas apropriadamente, sem nenhum integrante ficar com uma carga de atividades muito grande ou eventualmente muito pequena. Depois que os grupos são formados, os temas são escolhidos num prazo que varia de duas a três semanas e um professor é designado como orientador do trabalho, tendo-se em conta sua disponibilidade e seu campo de competência.

A segunda questão, ligada à avaliação do TCC, é delicada e muitas vezes polêmica. Isso acontece em virtude da subjetividade de avaliação. Por mais que os critérios sejam bem definidos e detalhados, pode haver discrepância entre a nota do avaliador do trabalho e a nota do orientador do TCC. Nesses casos, o trabalho passa por uma terceira avaliação, a fim de dirimir o impasse.

Outro ponto a ser ressaltado na avaliação é a manutenção do mesmo avaliador desde a proposta inicial do trabalho até a apresentação final, que antecede o INOVAFEI. Dessa maneira, o avaliador pode ver claramente se o projeto está evoluindo de modo satisfatório ao longo das disciplinas TCC1 e TCC2.

Na Tabela 1 são apresentadas as ponderações utilizadas para avaliar os Trabalhos de Conclusão de Curso. Com base em reuniões semanais e na participação, os professores que ministram as disciplinas de TCC 1 e TCC 2 atribuem uma nota que equivale a $10 \%$ do total. Ao orientador do projeto cabe um peso de $15 \%$ na avaliação, tendo-se em conta a presença do grupo em reuniões e também a qualidade do trabalho escrito gerado. Já o avaliador do projeto é responsável por $75 \%$ da nota total, julgando tanto o trabalho escrito como as apresentações orais dos estudantes. É extremamente importante que os egressos do curso de Engenharia Química desenvolvam habilidade de comunicação escrita, bem como oral.

Tabela 1 - Ponderações nas avaliações dos TCCs

\begin{tabular}{ccc}
\hline parecer & tipo de avaliação & ponderação \\
\hline professor da disciplina & reuniões & $10 \%$ \\
orientador & reuniões / trabalho escrito & $15 \%$ \\
avaliador & trabalho escrito & $50 \%$ \\
avaliador & apresentação oral & $25 \%$ \\
\hline
\end{tabular}

Sobre as habilidades citadas no parágrafo anterior, cabe aqui um comentário, resultado de muitos anos de observação das atividades de TCC. Na grande maioria das vezes, os alunos possuem uma enorme facilidade para as apresentações orais. Eles falam com desenvoltura e segurança, conseguindo sintetizar uma grande quantidade de informações em quinze minutos. Além disso, durante a arguição do trabalho, defendem seus pontos de vista com veemência e pouca ou eventualmente nenhuma hesitação. Contudo, a habilidade escrita é o calcanhar de Aquiles da maioria. Normalmente, os textos produzidos pelos estudantes são rebuscados, mal estruturados e confusos, o que é perfeitamente compreensível, tendo-se em mente a vivência profissional ainda limitada desses jovens. Nesse aspecto, os docentes podem proporcionar um 
ganho enorme na formação dos alunos, por meio de correção detalhada da parte escrita, com trabalho árduo, envolvendo muitas idas e vindas.

\section{O INOVAFEI E OS TCCS DE ENGENHARIA QUÍMICA}

Os TCCs de Engenharia Química foram implantados em 2005, muito antes da criação do INOVAFEI, que ocorreu somente em 2013. Contudo, o INOVAFEI deu muito mais visibilidade aos projetos de conclusão dos estudantes de Engenharia Química, por reunir cursos de diversas áreas e atrair a atenção de um público mais amplo, com representantes de indústrias de diversos segmentos. Na última edição do INOVAFEI, em dezembro de 2016, houve a apresentação de 11 TCCs da Engenharia Química.

Os temas abordados nesses trabalhos são bastante variados, mas é possível classificálos em oito áreas diferentes, como indicado na Tabela 2. Essa classificação é a mesma que foi utilizada no Congresso Brasileiro de Engenharia Química, em 2016.

Tabela 2 - Classificação dos temas de TCCs

\begin{tabular}{cc}
\hline sigla & área \\
\hline AMB & Engenharia Ambiental e Tecnologias Limpas \\
BIO & Processos Biotecnológicos \\
CAT & Engenharia das Reações Químicas e Catálise \\
EPG & Energia, Petróleo, Gás e Biocombustíveis \\
FEN & Fenômenos de Transporte e Sistemas Particulados \\
MAT & Engenharia de Materiais e Nanotecnologia \\
SIM & Simulação, Otimização e Controle de Processos \\
TER & Engenharia das Separações e Termodinâmica \\
\hline
\end{tabular}

Na Tabela 3 se faz uma compilação da distribuição dos TCCs de acordo com o tema, desde a implantação do INOVAFEI, no segundo semestre de 2013. Percebe-se a predominância da área de Engenharia Ambiental e Tecnologias Limpas, com 19 trabalhos ao longo desse tempo. Trata-se de um dado relevante, mostrando que os estudantes têm preocupação com o campo em questão. Esses trabalhos geralmente contemplam a parte de tratamento de efluentes e projetos de reciclagem e reaproveitamento de resíduos.

Tabela 3 - Temas dos TCCs da Engenharia Química

\begin{tabular}{ccccccccc}
\hline área & $\mathbf{2}^{\mathbf{0}} / \mathbf{1 3}$ & $\mathbf{1}^{\mathbf{0}} / \mathbf{1 4}$ & $\mathbf{2}^{\mathbf{0}} / \mathbf{1 4}$ & $\mathbf{1}^{\mathbf{0}} / \mathbf{1 5}$ & $\mathbf{2}^{\mathbf{0}} / \mathbf{1 5}$ & $\mathbf{1}^{\mathbf{0}} / \mathbf{1 6}$ & $\mathbf{2}^{\mathbf{0}} \mathbf{1 6}$ & total \\
\hline AMB & 4 & 2 & 1 & 1 & 3 & 2 & 6 & 19 \\
BIO & 2 & 0 & 0 & 0 & 1 & 0 & 1 & 4 \\
CAT & 1 & 0 & 0 & 2 & 0 & 0 & 0 & 3 \\
EPG & 0 & 0 & 2 & 1 & 1 & 1 & 0 & 5 \\
FEN & 1 & 0 & 0 & 0 & 0 & 0 & 1 & 2 \\
MAT & 0 & 1 & 1 & 0 & 0 & 0 & 2 & 4 \\
SIM & 1 & 2 & 3 & 2 & 1 & 2 & 1 & 12 \\
TER & 0 & 0 & 0 & 0 & 1 & 1 & 0 & 2 \\
total & 9 & 5 & 7 & 6 & 7 & 6 & 11 & 51 \\
\hline
\end{tabular}

Outra área que se destaca na distribuição de temas na tabela anterior é a de Simulação, 
Otimização e Controle de Processos, com um total de 12 TCCs. Isso se explica pelo fato de muitos estudantes não terem afinidade com trabalhos experimentais, preferindo mais a área computacional. Outra justificativa é que o uso de um software é muito mais flexível do que o uso dos laboratórios: os estudantes podem realizar as atividades em seus próprios computadores e nos momentos que forem mais convenientes. Isso é chave para aqueles que fazem estágio no último ano do curso. Entretanto, independentemente disso, os laboratórios do Centro Universitário da FEI ficam abertos em horários flexíveis, de segunda a sexta-feira, nos três períodos, e aos sábados, até o final da tarde, para os estudantes que quiserem se dedicar a temas de caráter experimental.

Um último ponto a ser citado sobre os TCCs da Engenharia Química e o INOVAFEI concerne os trabalhos que de fato serão apresentados nesse evento. Essa escolha é feita pela Comissão do TCC, que delibera sobre quais trabalhos têm mérito técnico / científico / inovador para ser divulgado. De todos os trabalhos escolhidos e posteriormente apresentados no INOVAFEI, dois são selecionados por uma banca de profissionais externos. Esses trabalhos são então premiados no final do evento. O ponto positivo dessa estratégia é que isso acaba gerando uma competição saudável entre os grupos: todos querem fazer um bom trabalho e uma excelente apresentação, mesmo com o prêmio não envolvendo quaisquer vantagens materiais.

\section{CONCLUSÕES}

Nesse artigo se faz uma análise da forma de se conduzir os TCCs no curso de Engenharia Química do Centro Universitário da FEI. Falou-se também a respeito do INOVAFEI, evento que reúne os TCCs de diversas áreas diferentes da escola. Essa análise indicou que os TCCs devem ter caráter de integração de conhecimentos dentro da Engenharia Química e serem realizados não individualmente, mas sim em grupos compostos por pelo menos quatro alunos. Deve-se lembrar que a formação de um engenheiro não é meramente técnica, ela contempla também competências comportamentais. Desde a criação do INOVAFEI, em 2013, houve a realização de 51 trabalhos. Uma compilação dos dados de distribuição de temas dos TCCs (Tabela 3) mostrou a preocupação dos estudantes com a área ambiental e a preferência por realizar trabalhos computacionais.

Outra conclusão importante sobre os TCCs é a manutenção de um mesmo avaliador durante todo o desenvolvimento do trabalho, com o intuito de se verificar sua evolução. Além disso, a maior exigência que os docentes possuem é a de promover uma melhora na habilidade de escrita dos estudantes. Além do próprio avaliador, que tem peso maior nas notas de TCC, há o parecer dos professores das disciplinas TCC1 e TCC2, bem como o julgamento do próprio orientador, que participa mais ativamente do trabalho (Tabela 1). Concluiu-se que esse processo de avaliação funciona bem, sendo pouco frequentes as discrepâncias de notas entre os pareceres.

O fato de o INOVAFEI oferecer premiação para os dois melhores trabalhos de cada área faz com que se desenvolva um ambiente de competição saudável, no qual os estudantes se orgulham e defendem seus projetos. 


\title{
5. AGRADECIMENTOS
}

Ao Centro Universitário FEI pelo suporte para o desenvolvimento de todos os trabalhos apresentados no INOVAFEI.

\section{REFERÊNCIAS}

d'ANGELO, J.V.H; ZEMP, R.J.; RODRIGUES, M.T.M.; RAVAGNANI, S.P. Trabalho de Conclusão de Curso - a experiência da Faculdade de Engenharia Química da Unicamp. Revista Ensino Superior Unicamp, v.8, 2013

Congresso Brasileiro de Engenharia Química 2016. Programação Científica. Disponível em http://cobeq2016.com.br/br/node/1557

Conselho Nacional de Educação; Camâra de Edução Superior. Resolução CNE/CES 11/2002. Disponível em http://portal.mec.gov.br/conselho-nacional-deeducacao/apresentacao

COSTA, E.; MEIRA, M. Escrevendo Trabalhos de Conclusão de Curso. Rio de Janeiro: Editora Ciência Moderna, 2012

GLOBAL ENTREPENEURSHIP MONITOR; MACEDO, M. M. [et al.] Empreendedorismo no Brasil: 2015. Curitiba: IBQP, 2015

MARTINS JR., J. Como escrever trabalhos de conclusão de curso. Rio de Janeiro: Editora Vozes, 2008

\section{INOVAFEI AND THE CHEMICAL ENGINEERING'S FINAL TERM PAPERS AT FEI UNIVERSITY}

\author{
R. B. TÔRRES ${ }^{1}$, I. C. FRANCO ${ }^{1}$, L. F. NOVAZZ1 ${ }^{1, *}$ \\ ${ }^{1}$ FEI University, Department of Chemical Engineering \\ *E-mail: lnovazzi@fei.edu.br
}

\begin{abstract}
The INOVAFEI was established at FEI University in 2013 and is a biannual event where undergraduate Final Term Papers of many courses are presented. These works, which involve integration of different branches in Chemical Engineering as well as related areas, are developed in one year and guided by an advisor. In this paper, the conduction of Final Term Papers is described. Students are exhorted to work in groups, instead of a standalone basis, so they can improve important personal skills, such as teamwork. A professor is assigned and evaluates the work from the beginning until the end of the project. Since INOVAFEI has been created, 51 works were carried out, and most of them connected to waste treatment and clean technologies. The two best projects in INOVAFEI are awarded, creating a healthy competitive environment among students.
\end{abstract}

KEYWORDS: Final Term Paper; INOVAFEI; Chemical Engineering; education. 